Review Article

\title{
Efficacy and Safety of Guizhi Decoction Associated Formulas for Allergic Rhinitis: A Systematic Review
}

\author{
Shasha Yang, ${ }^{1}$ Qinwei Fu $\mathbb{D},{ }^{2}$ Hua Deng, ${ }^{1}$ Jing Wu, ${ }^{3}$ Qinxiu Zhang $\mathbb{D}^{2,4}$ Limin Wang, \\ and Xianfeng Yao ${ }^{1}$ \\ ${ }^{1}$ The First Affiliated Hospital of Guizhou University of Traditional Chinese Medicine, Guiyang 550002, China \\ ${ }^{2}$ Hospital of Chengdu University of Traditional Chinese Medicine, Chengdu University of Traditional Chinese Medicine, \\ Chengdu 610072, China \\ ${ }^{3}$ Guizhou University of Traditional Chinese Medicine, Guiyang 550025, China \\ ${ }^{4}$ School of Medical and Life Sciences/Reproductive \& Women-Children Hospital, \\ Chengdu University of Traditional Chinese Medicine, Chengdu 610041, China
}

Correspondence should be addressed to Qinxiu Zhang; zhqinxiu@163.com

Received 17 May 2020; Accepted 4 January 2021; Published 13 January 2021

Academic Editor: Roberto Miniero

Copyright (c) 2021 Shasha Yang et al. This is an open access article distributed under the Creative Commons Attribution License, which permits unrestricted use, distribution, and reproduction in any medium, provided the original work is properly cited.

In this study, we aim to evaluate the efficacy and safety of Guizhi Decoction associated formulas for the treatment of allergic rhinitis. A total of seven online databases were searched to collect studies published up to Feb $23^{\text {rd }}, 2020$. Study quality of each included article was evaluated by the Cochrane Collaboration risk of bias tool. Systematic reviews were conducted based on the Cochrane systematic review method by using RevMan 5.3 Software. Among the included trials, Guizhi Decoction associated formulas alone (or plus Western medicine, or acupoint-based therapy) were main therapies in experimental groups. Interventions in control groups include Western medicine, Guizhi Decoction associated formulas alone, Chinese patent medicine, and placebo control. Primary outcomes in this study include recovery rate, accumulative marked effective rate, accumulative effective rate, and recurrence rate. Finally, 23 trials involving 2281 participants were included. Results of systematic reviews show that Guizhi Decoction and associated formulas alone, plus Western medicine or plus acupoint-based therapies, were significantly better compared with using Western medicine alone in terms of efficacy. In addition, the formulas plus nasal TCM fumigation therapy could improve effective rate for AR treatment compared to using the formulas alone. More types and cases of adverse events were reported in the control groups (Western medicine alone), but events of included trials were all mild and did not need specific medical intervention. More RCTs of high quality, and large sample size, with appropriate blinding methods or nonblinded pragmatic trials of Guizhi Decoction and associated formulas for AR are needed.

\section{Introduction}

As one of the most common allergic diseases, allergic rhinitis (AR) can lead to symptoms including nasal sneezing, discharge, congestion, and itching, which may reduce patients' sleep quality, work productivity, and general functioning, thus often making them feel depressed and anxious $[1,2]$. Patients with AR develop specific immunoglobulin $E$ (IgE) antibody responses to indoor or outdoor environmental allergens, such as mites, pollen, house dust, animal dander, and mixed fungi, with exposure over time [3].
It was estimated that $20 \%$ to $40 \%$ of the population in the US and $10 \%$ to $20 \%$ of world population were suffering from $\mathrm{AR}[4,5]$, with medical and hidden burden. Common therapies for AR include intranasal antihistamines, and novel methods of delivery for intranasal steroids [6]; however, some adverse events including minor nose bleeding and sedation or impairment of psychomotor function were reported $[7,8]$.

As a classic and important series of TCM decoctions, Guizhi Decoction and associated formulas have been used for over 1,800 years in China and are widely applied for AR 
till now. The efficacy of Guizhi Decoction and associated formulas matches the pathogenesis of AR according to TCM theory, and research showed that Guizhi Decoction associated formulas could inhibit cholinergic transdifferentiation of sympathetic nerves and improve the anatomical and functional denervation of sympathetic nerves [9]. However, the use of Guizhi Decoction and associated formulas in the treatment of AR beyond China is not popular, and the clinical efficacy and safety of Guizhi Decoction and associated formulas plus other treatments such as Western medicine and acupoint-based therapies were not certain.

Several reviews concerning TCM therapies especially herbal medicine decoctions for AR have been published $[10,11]$, while no study on efficacy and safety of Guizhi Decoction and associated formulas for AR has been conducted. The aim of this systematic review is to identify the clinical efficacy and safety of Guizhi Decoction associated formulas for the treatment of AR and to compare the efficacy and adverse effects in control groups by several comparisons.

\section{Materials and Methods}

2.1. Protocol and Registration. This systematic review was registered in PROSPERO, an international prospective register of systematic reviews, with the registration number CRD42020163034 (available from https://www.crd.york.ac. uk/prospero/display_record.php?RecordID=163034).

2.2. Search Strategy. We searched seven electronic databases, including Embase, PubMed, Cochrane Library, the China National Knowledge Infrastructure (CNKI), Technology Periodical Database (VIP), Wanfang Data Information Site, and SinoMed $(\mathrm{CBM})$ up to Feb $23^{\text {rd }}, 2020$. The search strategy and inclusion criteria were decided according to the guidance of the PRISMA agreement [12]. We used the following two groups of search terms in English: (1) "allergic rhinitis", "AR", "anaphylactic rhinitis" connected with "OR"; (2) "Guizhi", "Gui Zhi”, "Cassia twig", "Ramulus Cinnamomi" connected with "OR". The above search terms of (1) and (2) were connected with term "AND". All searches were limited to trials of RCT in humans and were conducted in electronic databases by two authors independently. We also searched with related search terms in Chinese and searched the references of the original and review articles manually for possible related trials and also tried to get grey literatures identified through other sources. Detailed search strategies are in file S1.

2.3. Inclusion Criteria. In this systematic review, we searched and included trials according to the following criteria:

(1) Trials including participants that were diagnosed with AR according to certain published guidelines with signs, symptoms, and laboratory findings.

(2) Prospective randomized controlled trials (RCTs).

(3) Trials in which Guizhi Decoction and associated formulas alone or plus other therapy(ies) were applied in experimental groups. The patients in control groups received conventional therapy(ies), other TCM therapies, or placebo regimens. Trials for more than one disease (AR) were excluded, such as asthma, cough, or conjunctivitis.

(4) Efficacy was evaluated according to certain published guidelines. Primary outcomes included recovery rate, accumulative marked effective rate, accumulative effective rate, and recurrence rate. Accumulative effective rate is the sum of recovery rate (if reported), marked effective rate (if reported), and effective rate (if reported), and accumulative marked effective rate is the sum of the first and the second one.

(5) Trials in Chinese or English.

2.4. Study Selection and Data Extraction. According to the above design, two reviewers (Qinwei Fu and Hua Deng) searched the online databases listed above and recorded the titles and abstracts of all the articles. Three evaluators (Limin Wang, Hua Deng, and Jing Wu) assessed the eligibility of these articles and made decisions on every research (inclusion or exclusion) independently. If they did not reach the same decision, the concerned articles were discussed with a fourth reviewer (Qinxiu Zhang). Three reviewers (Shasha Yang, Jing $\mathrm{Wu}$, and Xianfeng Yao) extracted data independently from each study. Differences of extracted data were solved after discussion with a fourth reviewer (Qinxiu Zhang).

2.5. Quality Assessment. Quality assessment of all the trials included in this review was independently evaluated by three reviewers (Shasha Yang, Qinwei $\mathrm{Fu}$, and Jing $\mathrm{Wu}$ ) using the Cochrane Collaboration risk of bias tool by RevMan 5.3 Software. Any disagreement was resolved by discussions with a fourth reviewer (Qinxiu Zhang).

2.6. Statistical Analysis. This systematic review was performed with the RevMan 5.3. For outcome measures, the overall effect sizes were determined as the mean difference (MD) for continuous outcomes, and RR for binary outcomes with their 95\% confidence intervals (CI), with $P<0.05$ indicating significant differences for effect sizes. The $Q$ and I2 test statistics were conducted to examine heterogeneity, with I $>50 \%$ indicating significant heterogeneity. Fixedeffects model was applied to statistical analysis. If the heterogeneity was still obvious (I2 > 50\%) and more than six trials were included, then sensitivity analysis and subgroup analysis were conducted to identify certain variables or (and) trials leading to high heterogeneity. Exploration of publication bias by funnel plots was planned if more than six trials were included.

\section{Results}

3.1. Study Inclusion. Initially, 668 records were searched from seven databases with no grey literature reference. After 
the removal of duplicates, the records were decreased to 195 . Based on titles and abstracts of records, we excluded 67 papers with reasons such as case reports, observational studies, uncontrolled studies, animal experiments, reviews, and studies with no randomization-control design and not related to Guizhi Decoction associated formulas for AR. The remaining 128 articles were downloaded for further selection, and 105 articles were excluded with reasons. Eventually, 23 trials of 22 studies (one three-arm study was recombined to two trials for comparison) were included [13-34] (Figure S1).

3.2. Study Characteristics. All 23 included RCTs were conducted in China and published in Chinese. In total, 2281 participants aging from 8 to 71 with AR from 50 days to 27 years were involved in 23 RCTs. Baseline characteristics were not detailed in several trials, but no significant difference among them between experimental and control groups $(P<0.05)$ was mentioned in all of the studies. As for the interventions of experimental groups, Guizhi Decoction associated formulas alone, combined with other TCM decoction/powder, with acupoint-based therapy (e.g., acupuncture, moxibustion, acupoint application, or auricular point pressing), with Western medicine (the same as the medicine used in the control groups mostly), or with Chinese patent medicine, were applied mainly. Although some of the specific prescriptions of Guizhi Decoction associated formulas in this study were different among the included trials, most of them have the effect of promoting $Q i$ transmission and balancing Yin and Yang according to the theory of TCM and are widely used in the treatment of AR and many other diseases. As a result, they were considered as the same orientation. In addition, Western medicine alone was applied in control groups of 18 trials [13-16, 18, 19, 21-24, 26-28, 30-34], placebo control (normal saline, oral) in one trial [16], oral TCM decoction or powder, or Chinese patent medicine alone in two trials $[16,17]$, Chinese patent medicine combined with Western medicine in one trial [20], and Guizhi Decoction associated formulas plus TCM fumigation in one trial [29]. Detailed characteristics of the included trials are listed in Table S1.

3.3. Assessment of Quality and Bias. According to the results of Cochrane Collaboration risk of bias tool [35], the method of randomization was described clearly and appropriately in eight trials $[15,17,21,22,27,28,31,34]$ with no trial in high risk of bias. Two trials described the method of allocation concealment clearly $[17,33]$ while others were described unclearly. No trial reported blinding method in addition to two studies with specially assigned personnel in outcome assessment blinding $[15,33]$. The bias for each trial is shown in Figure 1, and the bias summary is shown in Figure 2.

\subsection{Efficacy of Guizhi Decoction Associated Formulas in AR Patients}

3.4.1. Guizhi Decoction Associated Formulas versus Western Medicine. Compared with the Western medicine groups, significant improvement was found in Guizhi Decoction associated formulas groups, including recovery rate without heterogeneity in five trials $(\mathrm{RR}=1.67 ; \mathrm{P}$ for $\mathrm{RR}<0.01 ; 95 \%$ CI: $\left.1.34-2.08 ; \mathrm{I}^{2}=0 \%\right)[13,23,25,27,28]$, accumulative marked effective rate without heterogeneity in nine trials $(\mathrm{RR}=1.73 ; \mathrm{P}$ for $\mathrm{RR}<0.01 ; 95 \% \mathrm{CI}$ : $\left.1.47-2.02 ; \mathrm{I}^{2}=0 \%\right)[13,16,18,23,26-28,30,33]$, and accumulative effective rate with mild heterogeneity in nine trials $(\mathrm{RR}=1.20$; P for $\mathrm{RR}<0.01$; $95 \% \mathrm{CI}$ : 1.13-1.27; $\left.\mathrm{I}^{2}=0 \%\right)[13,16,18,23,26-28,30,33]$ (Table 1, Figures $\mathrm{S} 2$ and S3).

In addition, the patients with Guizhi Decoction associated formulas were reported with significantly lower recurrence rate in four trials compared with the control groups after both three months $(\mathrm{RR}=0.14$; $\mathrm{P}$ for $\mathrm{RR}<0.01 ; 95 \% \mathrm{CI}$ : $\left.0.04-0.47 ; \mathrm{I}^{2}=30 \%\right)$ and six months $(\mathrm{RR}=0.20 ; \mathrm{P}$ for RR < 0.01; 95\% CI: $\left.0.10-0.44 ; \mathrm{I}^{2}=34 \%\right)$ [13, 26-28]. Results of two trials also revealed that more cases with main symptoms disappeared in the experimental groups $\left(\mathrm{RR}=1.95 ; \mathrm{P}\right.$ for $\left.\mathrm{RR}=0.05 ; 95 \% \mathrm{CI}: 1.01-3.77 ; \mathrm{I}^{2}=79 \%\right)$ $[27,28]$. Subgroup analysis was conducted, while no obvious difference was observed between the two trials. However, the duration of allergic rhinitis was not reported in one of them, which might be the course of considerable heterogeneity [28] (Table 1, Figure S4).

\subsubsection{Guizhi Decoction Associated Formulas plus Western} Medicine versus Western Medicine. By comparison of the Guizhi Decoction associated formulas plus Western medicine groups versus Western medicine groups (Table 1, Figure S5), the pooled results favored the experimental groups on recovery rate without heterogeneity in two trials $\left(\mathrm{RR}=1.26 ; \mathrm{P}\right.$ for $\left.\mathrm{RR}=0.13 ; 95 \% \mathrm{CI}: 0.94-1.68 ; \mathrm{I}^{2}=0 \%\right)$ $[14,31]$, and on accumulative marked improvement rate $\left(\mathrm{RR}=1.13 ; \mathrm{P}\right.$ for $\left.\mathrm{RR}=0.13 ; 95 \% \mathrm{CI}: 0.96-1.33 ; \mathrm{I}^{2}=0 \%\right)$ and accumulative effective rate $(\mathrm{RR}=1.22$; $\mathrm{P}$ for $\mathrm{RR}<0.01 ; 95 \%$ CI: $\left.1.13-1.32 ; \mathrm{I}^{2}=0 \%\right)$ in four trials $[14,22,31,34]$.

3.4.3. Guizhi Decoction Associated Formulas plus AcupointBased Therapy versus Western Medicine. Results of systematic review showed that Guizhi Decoction associated formulas plus certain acupoint-based therapy, including acupuncture, normal moxibustion, heat-sensitive moxibustion, and acupoint application, could provide better improvement on recovery rate $(\mathrm{RR}=1.48$; $\mathrm{P}$ for $\mathrm{RR}=0.03$; 95\% CI: 1.03-2.12; $\mathrm{I}^{2}$ : not applicable) [21], on accumulative marked improvement rate $(\mathrm{RR}=1.07$; $\mathrm{P}$ for $\mathrm{RR}=0.89 ; 95 \%$ CI: $\left.0.39-2.93 ; \mathrm{I}^{2}=76 \%\right)[15,19]$, and on accumulative effective rate $(\mathrm{RR}=1.13$; $\mathrm{P}$ for $\mathrm{RR}=0.18$; $95 \% \mathrm{CI}$ : $0.95-1.35$; $\left.I^{2}=66 \%\right)[15,19,21]$. Subgroup analysis favored Guizhi Decoction associated formulas plus acupuncture with moxibustion compared with plus moxibustion or acupoint application on accumulative marked improvement rate and accumulative effective rate $[15,19,21]$ (Table 1, Figure S6).

3.4.4. Guizhi Decoction Associated Formulas versus Guizhi Decoction Associated Formulas plus TCM External Therapy. Control therapies of trials in this comparison include acupuncture, moxibustion, and local TCM fumigation. 
Efficacy and safety of Guizhi Decoction associated formulas for allergic rhinitis: a systematic review

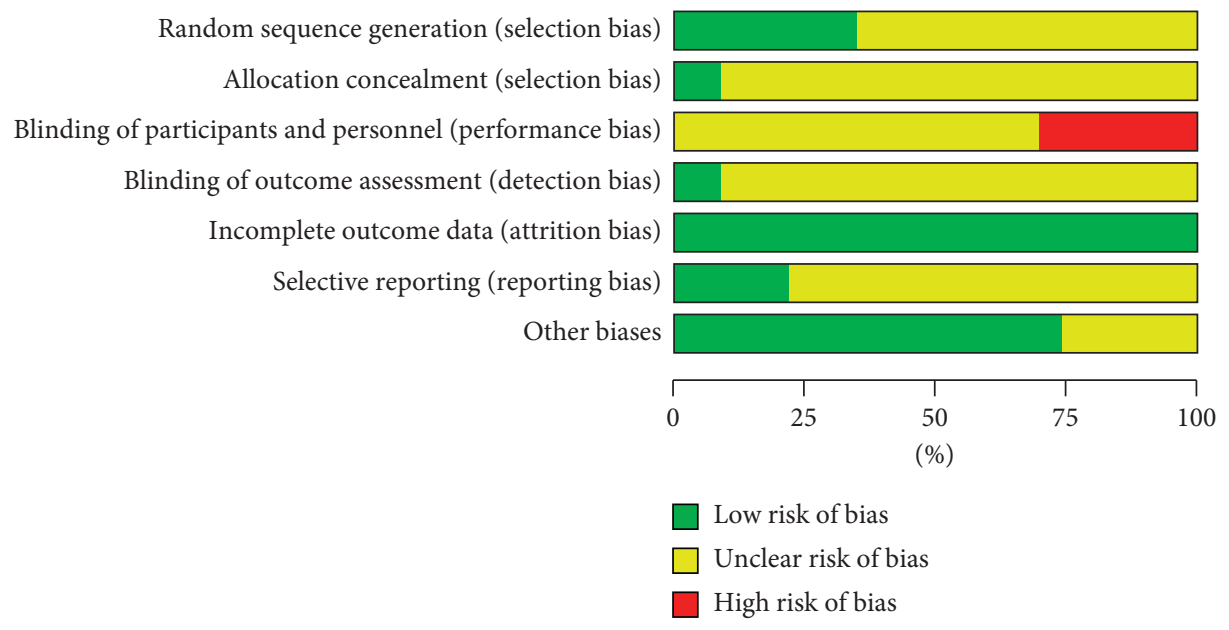

FIgURE 1: Risk of bias graph.

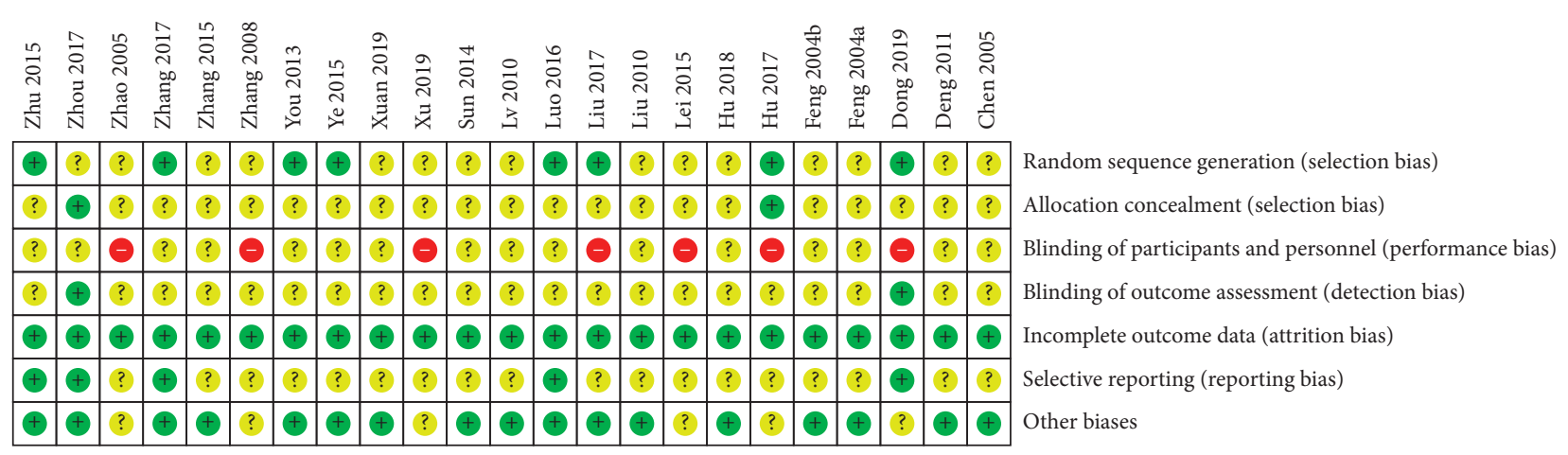

FIGURE 2: Risk of bias summary.

Compared with the experimental groups (Guizhi Decoction associated formulas), pooled results of two trials showed an increase in recovery rate $(\mathrm{RR}=0.78$; $\mathrm{P}$ for $\mathrm{RR}=0.04 ; 95 \% \mathrm{CI}$ : $\left.0.61-0.99 ; \mathrm{I}^{2}=0 \%\right)$ and a significant improvement on accumulative effective rate $(\mathrm{RR}=0.82$; $\mathrm{P}$ for $\mathrm{RR}=0.01 ; 95 \% \mathrm{CI}$ : $\left.0.71-0.96 ; \mathrm{I}^{2}=55 \%\right)$. In consideration of control therapies, subgroup analysis favored Guizhi Decoction associated formulas plus acupuncture with moxibustion compared with oral administration and nasal fumigation of Guizhi Decoction associated formulas on accumulative effective rate $[17,29]$ (Table 1, Figure S7).

3.5. Adverse Events Reported in Trials. Adverse events on the experimental groups were reported in four trials, and two trials reported no adverse event. For the experimental groups with Guizhi Decoction associated formulas applied, such events included palpitation (1 case), thirst (1 case), stomachache (1 case), and diarrhea (1 case) among the two studies covering 100 patients $[33,34]$. Local burns recovered without scar after the Safflower Oil smeared (2 cases) and bucking (because of the smoke) relieved with a mask wore (1 case) were reported in one trial covering 30 patients, on whom heat-sensitive moxibustion plus Guizhi Decoction associated formulas were applied [15]. Liu and colleagues
(Guizhi Decoction associated formulas plus acupoint application) mentioned adverse event as 5\%, but with no details reported [21].

However, events were found in the control groups of all the six trials reported $[15,20,21,28,33,34]$, including drowsiness (13), fatigue (2), thirst (2), stomachache (3), and diarrhea (2) among the 100 patients in two trials $[33,34]$. In addition, five cases ( 5 of 30) of slight adverse events (such as drowsiness, fatigue, and thirst) were reported in Dong 2019 [15], and Lin and colleagues found that nearly all the patients in control groups manifested fatigue, drowsiness, inattention, thirst, and weakness, with several cases of stomach discomfort [20]. Two trials mentioned adverse event as $4 \%$, but also with no details reported [21, 28].

The adverse events reported in the experimental and control groups were all mild, and relieved or gone, or did not require specific intervention or medical evaluation. Events were not reported in the other 17 trials.

\section{Discussion}

To the best of our knowledge, this is the first systematic review of Guizhi Decoction associated formulas for AR. Guizhi Decoction is "the first and most applied decoction" in the Treatise on Febrile Disease, a TCM classic and regarded 
TABLE 1: Summary of findings.

\begin{tabular}{|c|c|c|c|c|c|c|c|}
\hline Intervention & Outcome & $\begin{array}{l}\text { No. of } \\
\text { trials }\end{array}$ & $\begin{array}{c}\text { Participants (E: male/ } \\
\text { female }) /(\mathrm{C}: \text { male/female })\end{array}$ & $\begin{array}{l}\text { Effect size } \\
\quad(\mathrm{RR})\end{array}$ & $95 \% \mathrm{CI}$ & $\begin{array}{l}P \text { value Of } \\
\text { effect size }\end{array}$ & $\begin{array}{c}\mathrm{I}^{2} \\
\text { value }\end{array}$ \\
\hline \multirow{7}{*}{ GZDAF vs. WM } & Recovery rate & 5 & $(154 / 109) /(138 / 99)$ & 1.67 & $\begin{array}{l}1.34 \text { to } \\
2.08\end{array}$ & \multirow{5}{*}{$<0.01$} & $0 \%$ \\
\hline & $\begin{array}{c}\text { Accumulative marked effective } \\
\text { rate }\end{array}$ & \multirow{2}{*}{9} & \multirow{2}{*}{$(269 / 192) /(247 / 183)$} & 1.73 & $\begin{array}{l}1.47 \text { to } \\
2.02\end{array}$ & & $0 \%$ \\
\hline & Accumulative marked rate & & & 1.20 & $\begin{array}{l}1.13 \text { to } \\
1.27\end{array}$ & & $28 \%$ \\
\hline & Recurrence rate (3 months) & \multirow[b]{2}{*}{4} & \multirow[b]{2}{*}{$(125 / 110) /(83 / 72)$} & 0.14 & $\begin{array}{c}0.04 \text { to } \\
0.47\end{array}$ & & $30 \%$ \\
\hline & Recurrence rate (6 months) & & & 0.20 & $\begin{array}{c}0.10 \text { to } \\
0.44\end{array}$ & & $34 \%$ \\
\hline & $\begin{array}{l}\text { Cases of main symptoms } \\
\text { disappearance (subgroup 1) }\end{array}$ & 1 & $(18 / 14) /(20 / 12)$ & 1.44 & $\begin{array}{l}1.02 \text { to } \\
2.05\end{array}$ & 0.05 & NA \\
\hline & $\begin{array}{c}\text { Cases of main symptoms } \\
\text { disappearance (subgroup 2) }\end{array}$ & 1 & $(21 / 19) /(25 / 15)$ & 2.75 & $\begin{array}{c}1.68 \text { to } \\
4.51\end{array}$ & $<0.01$ & NA \\
\hline \multirow{3}{*}{ GZDAF + WM vs. WM } & Recovery rate & 2 & $\mathrm{~A} *$ & 1.26 & $\begin{array}{c}0.94 \text { to } \\
1.68\end{array}$ & 013 & \\
\hline & $\begin{array}{l}\text { Accumulative marked effective } \\
\text { rate }\end{array}$ & \multirow{2}{*}{4} & \multirow{2}{*}{$\mathrm{B} *$} & 1.13 & $\begin{array}{c}0.96 \text { to } \\
1.33\end{array}$ & 0.13 & $0 \%$ \\
\hline & Accumulative marked rate & & & 1.22 & $\begin{array}{c}1.13 \text { to } \\
1.32 \\
\end{array}$ & $<0.01$ & \\
\hline \multirow{5}{*}{ GZDAF + ABT vs. WM } & Recovery rate & 1 & $(52 / 48) /(27 / 23)$ & 1.48 & $\begin{array}{l}1.03 \text { to } \\
2.12\end{array}$ & 0.03 & NA \\
\hline & $\begin{array}{l}\text { Accumulative marked effective } \\
\text { rate (subgroup 1) }\end{array}$ & 1 & $(15 / 15) /(14 / 16)$ & 0.6 & $\begin{array}{c}0.25 \text { to } \\
1.44\end{array}$ & 0.25 & NA \\
\hline & $\begin{array}{l}\text { Accumulative marked effective } \\
\text { rate (subgroup 2) }\end{array}$ & 1 & $(20 / 20) /(21 / 19)$ & 1.67 & $\begin{array}{l}1.05 \text { to } \\
2.66\end{array}$ & 0.03 & NA \\
\hline & $\begin{array}{l}\text { Accumulative marked rate } \\
\text { (subgroup 1) }\end{array}$ & 2 & $(35 / 35) /(35 / 35)$ & 1.05 & $\begin{array}{c}0.94 \text { to } \\
1.17\end{array}$ & 0.38 & $0 \%$ \\
\hline & $\begin{array}{l}\text { Accumulative marked rate } \\
\text { (subgroup 2) }\end{array}$ & 1 & $(20 / 20) /(21 / 19)$ & 1.41 & $\begin{array}{c}1.12 \text { to } \\
1.77\end{array}$ & $<0.01$ & NA \\
\hline \multirow{3}{*}{$\begin{array}{l}\text { GZDAF vs. } \\
\text { GZDAF + TCMET }\end{array}$} & Recovery rate & 2 & $(67 / 95) /(60 / 82)$ & 0.78 & $\begin{array}{c}0.61 \text { to } \\
0.99\end{array}$ & 0.04 & $0 \%$ \\
\hline & $\begin{array}{l}\text { Accumulative marked rate } \\
\text { (subgroup 1) }\end{array}$ & 1 & $(43 / 77) /(38 / 62)$ & 0.77 & $\begin{array}{c}0.66 \text { to } \\
0.89\end{array}$ & $<0.01$ & NA \\
\hline & $\begin{array}{l}\text { Accumulative marked rate } \\
\text { (subgroup 2) }\end{array}$ & 1 & $(24 / 18) /(22 / 20)$ & 0.88 & $\begin{array}{c}0.77 \text { to } \\
1\end{array}$ & 0.05 & NA \\
\hline
\end{tabular}

E: experimental group; C: control group; GZDAF: Guizhi Decoction associated formulas; WM: Western medicine; ABT: acupoint-based therapy; TCMET: TCM external therapy; NA: not applicable; A*: 61 for male (E and C)/53 for female (E and C) in Deng 2011, and (33/34)/(31/26) in Zhang 2017; B*: 61 for male (E and C)/53 for female (E and C) in Deng 2011, 72 for male (E and C)/62 for female (E and C) in Luo 2016, and (59/38)/(54/53) in Zhang 2017 + Zhu 2015.

as one of the pioneers for TCM theory and practice. Originating from the late Eastern Han Dynasty in China about 1800 years ago, Guizhi Decoction and associated formulas are still widely used till now especially in China.

In TCM theory, AR falls into excess syndrome (stagnant heat of lung meridian, mainly) and deficiency syndrome (including lung yang deficiency, spleen Qi deficiency, and kidney yang deficiency, mainly) [36]. Guizhi Decoction is categorized as prescription for relieving exterior disorders in classification of prescriptions of TCM, while it is also widely recognized and applied in internal diseases through promoting Q $i$ transmission and balancing Yin and Yang. Some formulas were transformed from Guizhi Decoction for specific purposes such as severe Yang deficiency, heat fire, or depression of exterior Qi, but their main principles are associated closely. Modern researches show that Guizhi Decoction associated formulas could inhibit cholinergic transdifferentiation of sympathetic nerves and improve the anatomical and functional denervation of sympathetic nerves [9]. Meanwhile, the formulas are also widely used in many diseases of internal, external, gynecologic, and other diseases such as colds, febrile diseases, various perspiration, digestive system diseases, respiratory diseases, ENT disease, throat disease, nervous system disease, cardiac autonomic neuropathy, and bone and joint diseases [37].

Results of our study show that, compared with applying Western medicine alone, higher total effective rate (including recovery rate, accumulative marked effective rate, and accumulative effective rate), lower recurrence rate (3 months and 6 months), and more cases with main symptoms of AR disappeared were reported in experimental groups, including applying Guizhi Decoction and associated formulas alone, plus Western medicine or plus acupoint-based therapies. In 
addition, there were fewer types and cases of adverse events in experimental groups. Some types of the events were found in both experimental and control groups, including thirst, stomachache, and diarrhea, while others appeared in Western medicine groups (drowsiness and fatigue) or experimental groups (local burns and bucking when applying moxibustion) only. However, adverse events reported were all mild and relieved or gone without specific medical intervention.

In addition, one comparison favored the control group (Guizhi Decoction and associated formulas plus TCM external therapy) with higher recovery rate and accumulative effective rate than experimental groups (Guizhi Decoction and associated formulas alone).

Acupoint-based therapies, such as acupuncture, moxibustion, acupoint catgut embedding, acupressure, and acupoint application, are important components of TCM external therapies. In China, some of the therapies such as acupuncture, moxibustion, acupoint catgut embedding, and acupoint application have been widely used in AR for symptoms relieving, severity reduction, adverse events decreasing, and life quality improving [38-41]. Such therapies in our study include heatsensitive moxibustion, acupuncture, acupoint application, and auricular point pressing mainly, with TCM nasal fumigation in one study. It should be pointed out that some TCM therapies were effective for some allergic or respiratory diseases, including multiplex meridian interventions for asthma and Chinese herbal medicine for acute exacerbations of COPD, and for food allergy and eczema [42-44]. In addition, several studies of high quality in recent years approved that some acupoint-based therapies were not inferior to Western medicine in preventing and controlling some diseases, such as acupuncture and acupressure for cancer pain, acupuncture for chemotherapy-induced peripheral neuropathy symptoms, acupuncture for chronic stable angina, and acupressure combined with TCM footbath for diabetic peripheral neuropathy [45-48]. In addition, the therapies could reduce some medication intake, especially those that may have substantial addiction and adverse events.

As for study quality and risk of bias, all the 23 trials are RCTs, but placebo control was only employed in one study. Eight trials employed appropriate randomization method with clear statement, while it was in unclear risk of bias for the other 15 trials. Allocation concealment and blinding method were of unclear risk of bias in most trials. No study reported drop-out, and a protocol or registration ahead of experiment was only reported in two trials. As a result, double-blind, prospective, randomized, placebo-controlled trials of Guizhi Decoction and associated formulas as a treatment for AR are urgently need. In addition, concerning practical applicability and extrapolation in real-world situations, pragmatic trials without blinding have been suggested for achieving clinically relevant results in recent years $[49,50]$. This type of trials is especially appropriate for TCM researches on efficacy and safety, considering the complexity and flexibility of TCM therapies.

\section{Limitations}

There are several limitations in our study. Firstly, trials included were of moderate to substantial risk of bias, such as lack of reporting about details of random sequence generation, concealment, and blinding of participants, personnel, and outcome assessment quality. This may lead to low quality of the included studies. Secondly, there was a wide range of publishing year (2004 to 2019) among the trials, which may lead to potential bias when they are pooled due to different editions of guidelines and standards (especially in diagnosis and outcomes). Finally, conforming to the criteria, assessment of publication bias was inapplicable for no more than 10 trials included in each comparison. The GRADE evidence profiles (EP) of our results are in Tables S2-S5, and most of them are in low or even very low quality.

As a result, more RCTs of high quality and large sample size, with appropriate blinding methods or nonblinded pragmatic trials by real-world researches, are needed to further improve and update our study.

\section{Conclusion}

In general, this systematic review demonstrated that applying Guizhi Decoction and associated formulas alone, plus Western medicine or plus acupoint-based therapies, may be safer and more effective for the treatment of AR than taking Western medicine alone. And the formulas plus nasal TCM fumigation could improve effective rate for AR treatment than using the formulas alone. More types and cases of adverse events were reported in the control groups (Western medicine alone), but events of included trials were all mild and did not need specific medical intervention. There is an urgent need for RCTs of high quality and large sample size, with appropriate blinding methods or nonblinded pragmatic trials of Guizhi Decoction and associated formulas for AR.

\section{Abbreviations:}

TCM: Traditional Chinese medicine

RR: Risk ratio

MD: Mean difference

CI: Confidence interval.

\section{Data Availability}

The data used in the study are available upon request to the corresponding author.

\section{Disclosure}

Qinwe Fu Co-first Author.

\section{Conflicts of Interest}

The authors declare that they have no conflicts of interest.

\section{Authors' Contributions}

All the authors equally contributed to the study. SS Yang, QW Fu, QX Zhang, Jing W H, and Deng were involved in the conception and design; all of the authors were involved in analysis and interpretation of the data; SS Yang, QW Fu, Jing W, QX Zhang, XF Yao, and LM Wang were involved in 
the drafting of the paper or revising it critically for intellectual content. All authors agree to be accountable for all aspects of the work.

\section{Acknowledgments}

This project was jointly supported by National Natural Science Foundation of China (No. 81960821), International Standard Preparation and Revision Project of the World Federation of Chinese Medicine Societies-International Guidelines for the Diagnosis and Treatment of Allergic Rhinitis in Traditional Chinese Medicine (SCM NP 2020-0097), Doctoral Fund Project of the First Affiliated Hospital of Guizhou University of Traditional Chinese Medicine (GYZYYFY-BS-2019(01)), Youth Science and Technology Talent Growth Project of Guizhou Provincial Department of Education (Qian Jiao He KY [2017]170), Research Project of Science and Technology of Traditional Chinese Medicine and National Medicine of Guizhou Administration of Traditional Chinese Medicine (QZYY-2018-026), and Research Practice and Innovation Projects for University Students, Chengdu University of Traditional Chinese Medicine, 2020-2021 (ky-2021013).

\section{Supplementary Materials}

Detailed search strategies, characteristics of included trials, GRADE evidence profiles (EP) of our results, the flow diagram of the study selection process, and forest plots of the comparisons in our study are listed in supplementary materials document. (Supplementary Materials)

\section{References}

[1] R. Muñoz-Cano, P. Ribó, G. Araujo, E. Giralt, J. SanchezLopez, and A. Valero, "Severity of allergic rhinitis impacts sleep and anxiety: results from a large Spanish cohort," Clinical and Translational Allergy, vol. 8, p. 23, 2018.

[2] H. Y. Huang, L. Z. Zhang, Q. X. Zhang et al., "Study on the distribution of 8 TCM syndromes in 1216 children with allergic rhinitis in Chengdu area," Zhonghua Er Bi Yan Hou Tou Jing Wai Ke Za Zhi, vol. 54, no. 8, pp. 576-583, 2019, in Chinese.

[3] D. I. Bernstein, G. Schwartz, and J. A. Bernstein, "Allergic rhinitis," Immunology and Allergy Clinics of North America, vol. 36, no. 2, pp. 261-278, 2016.

[4] P. M. Salo, A. Calatroni, P. J. Gergen et al., "Allergy-related outcomes in relation to serum IgE: results from the national health and nutrition examination survey 2005-2006," Journal of Allergy and Clinical Immunology, vol. 127, no. 5, pp. 1226-1235, 2011.

[5] D. Poddighe, A. Licari, S. Caimmi, and G. L. Marseglia, "Sublingual immunotherapy for pediatric allergic rhinitis: the clinical evidence," World Journal of Clinical Pediatrics, vol. 5, no. 1, pp. 47-56, 2016.

[6] F. C. L. Hoyte and H. S. Nelson, "Recent advances in allergic rhinitis," F1000Research, vol. 7, p. 1333, 2018.

[7] R. J. Salib and P. H. Howarth, "Safety and tolerability profiles of intranasal antihistamines and intranasal corticosteroids in the treatment of allergic rhinitis," Drug Safety, vol. 26, no. 12, pp. 863-893, 2003.
[8] L. Y. Chong, K. Head, C. Hopkins, C. Philpott, A. G. Schilder, and M. J. Burton, "Intranasal steroids versus placebo or no intervention for chronic rhinosinusitis," The Cochrane Database of Systematic Reviews, vol. 4, p. CD011996, 2016.

[9] Y.-c. Wang, D.-f. Ma, P. Jiang et al., "Guizhi decoction (桂枝 汤) inhibits cholinergic transdifferentiation by regulating imbalance of NGF and LIF in salt-sensitive hypertensive heart failure rats," Chinese Journal of Integrative Medicine, vol. 26, no. 3, pp. 188-196, 2020.

[10] F. Zhou, L.-J. Yan, G.-Y. Yang, and J.-P. Liu, "Acupoint herbal patching for allergic rhinitis: a systematic review and metaanalysis of randomised controlled trials," Clinical Otolaryngology, vol. 40, no. 6, pp. 551-568, 2015.

[11] Z. Zheng, Z. Sun, X. Zhou, and Z. Zhou, "Efficacy of Chinese herbal medicine in treatment of allergic rhinitis in children: a meta-analysis of 19 randomized controlled trials," Journal of International Medical Research, vol. 46, no. 10, pp. 4006-4018, 2018.

[12] D. Moher, A. Liberati, J. Tetzlaff, and D. G. Altman, "The PRISMA group (2009), "preferred reporting items for systematic reviews and meta-analyses: the PRISMA statement," PLoS Medicine, vol. 6, Article ID e1000097, 2009.

[13] C. He, "Treatment of perennial allergic rhinitis by buqi gubiao yishen therapy: a clinical observation of 56 cases," Journal of New Chinese Medicine, vol. 37, no. 12, pp. 28-29, 2005, in Chinese.

[14] L. Deng, "Clinical analysis of Chaihu Guizhi Decoction in the treatment of allergic rhinitis," Nei Mongol Journal of Traditional Chinese Medicine, vol. 30, no. 06, pp. 24-25, 2011, in Chinese.

[15] W. Dong, "Efficacy observation of heat-sensitive moxibustion combined with guizhi decoction in the treatment of allergic rhinitis," Master's thesis, Guangzhou University of Chinese Medicine, Guangzhou, China, 2019, in Chinese.

[16] W. Feng, X. Wang, M. Zhang, Z. Wei, and Y. Fang, "Clinical studies on effect of allergic rhinitis oral liquid on perennial allergic rhinitis," Journal of Guangzhou University of Traditional Chinese Medicine, vol. 02, pp. 101-104, 2004, in Chinese.

[17] C. Hu, "Effect of Guizhi Pingfeng Decoction combined with acupuncture on 42 cases of allergic rhinitis," Nei Mongol Journal of Traditional Chinese Medicine, vol. 36, no. Z1, pp. 63-64, 2017, in Chinese.

[18] S. Hu, "Effect of huangqi guizhi wuwu decoction and cocklebur powder in the treatment of allergic rhinitis and its effect on immune function," Master's thesis, Fujian University of Traditional Chinese Medicine, Fuzhou, China, 2018, in Chinese.

[19] L. Lei, "Clinical study on the treatment of allergic rhinitis with Guizhi Pingfeng Decoction and acupuncture," Asia-Pacific Traditional Medicine, vol. 11, no. 08, pp. 120-121, 2015, in Chinese.

[20] L. Zhong, "Clinical observation on 30 cases of allergic rhinitis treated with Xinqi Guizhi Decoction and nuckear dairy injection," Journal of Sichuan of Traditional Medicine, vol. 28, no. 01, pp. 109-110, 2010, in Chinese.

[21] J. Liu and B. Zhao, "Efficacy observation of modified formula of Guizhi decoction and Cangerzi powder combined with acupoint application on allergic rhinitis," Shanxi Journal of Traditional Chinese Medicine, vol. 33, no. 04, pp. 19-20, 2017, in Chinese.

[22] T. Luo, Q. Wu, and N. Wang, "Curative effect of huangqi decoction added with Guizhi on allergic rhinitis of lung Qi 
weak and cold pattern," World Chinese Medicine, vol. 11, no. 12, pp. 2584-2587, 2016, in Chinese.

[23] X. Lv, "55 cases of allergic rhinitis of yang deficiency type treated by Guizhi and longgu muli decoction," Zhejiang Journal of Traditional Chinese Medicine, vol. 45, no. 8, p. 573, 2010, in Chinese.

[24] L. Sun, D. You, R. Zhu, S. Xu, and H. Wang, "Clinical effect of Guizhi Decoction on allergic rhinitis," Journal of New Chinese Medicine, vol. 46, no. 12, pp. 150-151, 2014, in Chinese.

[25] Y. Xu, "Efficacy of the Guizhi decoction plus auricular point on clinical symptoms in patients with allergic rhinitis," Clinical Journal of Chinese Medicine, vol. 11, no. 32, pp. 28-30, 2019, in Chinese.

[26] Z. Xuan, "Clinical observation on the treatment of allergic rhinitis with Guizhi decoction and mahuang fuzi xixin decoction," Journal of Practical Traditional Chinese Medicine, vol. 35, no. 09, pp. 1052-1053, 2019, in Chinese.

[27] J. Ye and J. Lin, "32 cases of allergic rhinitis treated with Guizhi decoction and mahuang fuzi xixin decoction," Fujian Journal of Traditional Chinese Medicine, vol. 46, no. 02, pp. 47-48, 2015, in Chinese.

[28] H. You, Y. Chen, K. Lu, and D. Luo, "Guizhi Decoction and Xingsu Powder in the treatment of 40 cases of allergic rhinitis," Journal of New Chinese Medicine, vol. 45, no. 08, pp. 132-133, 2013, in Chinese.

[29] M. Zhang, J. Liu, and Yi Liu, "Clinical analysis of 120 cases of allergic rhinitis treated with oral administration of traditional Chinese medicine and nasal fumigation," Journal of Practical Traditional Chinese Medicine, vol. 06, pp. 349-350, 2008, in Chinese.

[30] F. Zhang and P. Zhang, "Yupingfeng Powder combined with Guizhi Decoction in the treatment of 65 cases of allergic rhinitis," Journal of Practical Traditional Chinese Medicine, vol. 31, no. 06, p. 507, 2015, in Chinese.

[31] C. Zhang and X. Chen, "Efficacy of chaihu Guizhi decoction combined with cetirizine in the treatment of allergic rhinitis and influence on inflammatory cytokines," World Chinese Medicine, vol. 12, no. 09, pp. 2065-2068, 2017, in Chinese.

[32] H. Zhao and Y. Wang, "42 cases of allergic rhinitis treated with Guizhi Pingfeng Decoction and acupuncture," Shaanxi Journal of Traditional Chinese Medicine, vol. 03, pp. 229-230, 2005, in Chinese.

[33] X. Zhou, L. Zhong, J. Wang, J. Zhu, and L. Chen, "Effect of huangqi Guizhi wuwu decoction on allergic rhinitis with deficiency of lung and spleen Qi," World Clinical Drugs, vol. 38, no. 06, pp. 408-412, 2017, in Chinese.

[34] S. Zhu, "Chaihu guizhisoup joint cetirizine treatment allergic rhinitis randomized controlled study," Journal of Practical Traditional Chinese Internal Medicine, vol. 29, no. 12, pp. 122-124, 2015, in Chinese.

[35] J. P. T. Higgins and S. Green, Cochrane Handbook for Systematic Reviews of Interventions, John Wiley \& Sons, Inc., New York, NY, USA, 2013, http://www.cochrane-handbook. org.

[36] D. XiongD. Yan et al., 13th Five Year Plan "Teaching Materials of Traditional Chinese Medicine in General Higher Education; National Refined Teaching Materials of Traditional Chinese Medicine in General Higher education," Otolaryngology Of Traditional Chinese Medicine, pp. 105-107, Shanghai science and technology press, Shanghai, China, 2017, in Chinese.

[37] B. Feng, Y. T. Fang, and R. S. Xu, "Research progress in modern clinical application and mechanism of Guizhi decoction," Zhongguo Zhong Yao Za Zhi, vol. 43, no. 12, pp. 2442-2447, 2018, in Chinese.
[38] J. Zhang, Y. Zhang, X. Huang et al., "Different acupuncture therapies for allergic rhinitis: overview of systematic reviews and network meta-analysis," Evid Based Complement Alternat Med, vol. 8363027, 2020.

[39] L. Xiao, B. Li, Y. H. Du, J. Xiong, and X. Gao, "Systematic evaluation of the randomized controlled trials about acupuncture and moxibustion treatment of allergic rhinitis," Zhongguo Zhen Jiu, vol. 29, no. 6, pp. 512-516, 2009, in Chinese.

[40] X.-R. Li, Q.-X. Zhang, M. Liu et al., "Catgut implantation at acupoints for allergic rhinitis: a systematic review," Chinese Journal of Integrative Medicine, vol. 20, no. 3, pp. 235-240, 2014.

[41] J. Peng, X. Wu, J. Hu, Y. Fang, M. Zi, and B. Liu, "Influencing factors on efficacy of summer acupoint application treatment for allergic rhinitis: a retrospective study," Journal of Traditional Chinese Medicine, vol. 32, no. 3, pp. 377-381, 2012.

[42] M.-H. Wang, C. Chen, M.-L. Yeh, and J.-G. Lin, "Using traditional Chinese medicine to relieve asthma symptoms: a systematic review and meta-analysis," The American Journal of Chinese Medicine, vol. 47, no. 08, pp. 1659-1674, 2019.

[43] J. Li, H. Zhang, H. Ruan et al., "Effects of Chinese herbal medicine on acute exacerbations of COPD: a randomized, placebo-controlled study," International Journal of Chronic Obstructive Pulmonary Disease, vol. 15, no. 15, pp. 2901-2912, 2020.

[44] Z. Wang, Z. Z. Wang, J. Geliebter, R. Tiwari, and X. M. Li, "Traditional Chinese medicine for food allergy and eczema," Annals of Allergy, Asthma \& Immunology, vol. 10, no. 20, pp. S1081-S1206, 2020.

[45] Y. He, X. Guo, B. H. May et al., "Clinical evidence for association of acupuncture and acupressure with improved cancer pain: a systematic review and meta-analysis," JAMA Oncology, vol. 6, no. 2, pp. 271-278, 2019.

[46] T. Bao, S. Patil, C. Chen et al., "Effect of acupuncture vs sham procedure on chemotherapy-induced peripheral neuropathy symptoms: a randomized clinical trial," JAMA Network Open, vol. 3, no. 3, Article ID e200681, 2020.

[47] L. Zhao, D. Li, H. Zheng et al., "Acupuncture as adjunctive therapy for chronic stable Angina," JAMA Internal Medicine, vol. 179, no. 10, pp. 1388-1397, 2019.

[48] Q. Fu, H. Yang, L. Zhang et al., "Traditional Chinese medicine foot bath combined with acupoint massage for the treatment of diabetic peripheral neuropathy: a systematic review and meta-analysis of 31 RCTs," Diabetes/Metabolism Research and Reviews, vol. 36, no. 2, Article ID e3218, 2020.

[49] H. C. Sox and R. J. Lewis, "Pragmatic trials," JAMA, vol. 316, no. 11, pp. 1205-1206, 2016.

[50] I. Ford and J. Norrie, "Pragmatic trials," New England Journal of Medicine, vol. 375, no. 5, pp. 454-463, 2016. 